hep-th/0503198

PUPT-2157

NSF-KITP-05-18

\title{
All Split Helicity Tree-Level Gluon Amplitudes
}

\author{
Ruth Britto^, Bo Feng^, Radu Roiban», Marcus Spradlin^, Anastasia Volovich^ \\ - School of Natural Sciences, Institute for Advanced Study, Princeton NJ 08540 USA \\ $\diamond$ Department of Physics, Princeton University, Princeton, NJ 08544 USA
}

*Kavli Institute for Theoretical Physics, University of California, Santa Barbara, CA 93106 USA

\begin{abstract}
Recently a new recursion relation for tree-level gluon amplitudes in gauge theory has been discovered. We solve this recursion to obtain explicit formulas for the closed set of amplitudes with arbitrarily many positive and negative helicity gluons in a split helicity configuration. The solution admits a simple diagrammatic expansion in terms of 'zigzag' diagrams. We comment on generalizations of this result.
\end{abstract}

March 2005 


\section{Introduction}

Gluon scattering amplitudes are important for computing jet processes as backgrounds in hadron colliders. However, the number of Feynman diagrams required in calculating these amplitudes quickly exceeds practical bounds as the number of external gluons increases. Remarkably, the final form of these amplitudes is often far simpler than one would guess from the expansion in Feynman diagrams. At tree level, the first examples of such simplicity came in the work of Parke and Taylor [1], who found an elegant single-term expression for maximally helicity violating (MHV) amplitudes. This formula was proven in [2] using the Berends-Giele recursion [3].

In a remarkable paper [4] Witten found that gluon amplitudes are localized on certain curves in twistor space and proposed a twistor string theory capturing the properties of these amplitudes. This discovery enabled a deeper understanding of their structure [5, 6, [7, 8, 9, 10, 11, 12, 13, 14]. One particular approach [7] offered a new diagrammatic expansion of tree amplitudes in terms of MHV vertices. This was a great improvement over the number and computational complexity of Feynman diagrams.

Another development in understanding the structure of gauge theory amplitudes has come from reconsidering their analytic structure in the space of complexified momenta [15] and making use of the interplay between tree and loop amplitudes. The divergent behavior of the one-loop contribution to a scattering amplitude encodes the tree-level contribution [16, 17]. Following recent progress in computing $\mathcal{N}=4$ amplitudes at one-loop [18, 19, 20,21,22 using unitarity based methods [23,24,25, 26, 27,28] this singular behavior was used in [29,22,30] to derive new representations of tree amplitudes. In particular, a study of analytic structure revealed that one-loop $\mathcal{N}=4$ amplitudes could be expressed essentially as a sum of products of tree amplitudes times known functions [21]. From this result, combined with a new formula derived from the singular behavior relations, it was inferred [30] that one could express a tree amplitude of gluons in terms of pairs of tree amplitudes of gluons and adjoint fermions and scalars with fewer external legs. In [31, it was proposed that this inference could be modified to involve only gluon amplitudes. An explicit quadratic recursion relation was conjectured and was found to yield directly the most compact formulas for tree amplitudes known so far.

The recursion relation of [31] was first proven and properly understood in [32 by considering the analytic properties of amplitudes in the space of complexified momenta. It was also shown that the recursion could be generalized and applied to prove the validity 
of the MHV diagrams of [7]. More recently it has been found that the recursion relation can be translated into terms of twistor geometry [33], and there have been extensions to amplitudes with fermions [34,35] or gravitons [36, 37] and loop amplitudes [38].

In this paper we study tree-level gluons amplitudes in helicity configurations of the form $(--\cdots-++\cdots+)$ which we call 'split helicity amplitudes.' These amplitudes form a closed set under the recursion relation (as well as under collinear limits). Here we solve the recursion to derive a simple expression for any split helicity amplitude. For an amplitude with $q$ negative helicity gluons and $n-q$ positive helicity gluons, our expression has $\left(\begin{array}{l}n-4 \\ q-2\end{array}\right)$ terms. Each term has can be interpreted as a 'zigzag diagram' involving the cyclic arrangement of gluons on a closed curve.

It would be very interesting to understand whether amplitudes other than those with split helicity configurations can be captured by some generalization of these zigzag diagrams. It would also be interesting to investigate the relationship of these diagrams to the diagrams of twistor geometry, for which some related results have appeared in [33].

\section{Preliminaries}

It is convenient to write tree amplitudes of gluons in the spinor-helicity formalism. In four dimensions any null vector $p$ can be written as a bispinor, $p_{a \dot{a}}=\lambda_{a} \widetilde{\lambda}_{a}$, where $\lambda_{a}$ and $\widetilde{\lambda}_{\dot{a}}$ are spinors of positive and negative chirality respectively. The inner product of vectors can be written in terms of the natural inner product of spinors $\langle i j\rangle=\epsilon_{a b} \lambda_{i}^{a} \lambda_{j}^{b}$ and $\left[\begin{array}{ll}i & j\end{array}\right]=\epsilon_{\dot{a} \dot{b}} \widetilde{\lambda}_{i}^{\dot{a}} \tilde{\lambda}_{j}^{\dot{b}}$. إ We work with amplitudes of cyclically ordered gluons, and all momenta are directed outward. For further details and references, see [4, 39].

Sums of cyclically consecutive momenta will be denoted by

$$
P_{x, y} \equiv p_{x}+p_{x+1}+\cdots+p_{y}
$$

We define the products

$$
\begin{aligned}
\left\langle i\left|P_{x, y}\right| j\right] & \equiv \sum_{k=x}^{y}\langle i k\rangle[k j] \\
\left\langle i\left|P_{x_{1}, y_{1}} \cdots P_{x_{r}, y_{r}}\right| j\right\rangle & \equiv \sum_{k_{1}=x_{1}}^{y_{1}} \cdots \sum_{k_{r}=x_{r}}^{y_{r}}\left\langle i k_{1}\right\rangle\left[\begin{array}{lll}
k_{1} & k_{2}
\end{array}\right] \cdots\left\langle k_{r} j\right\rangle .
\end{aligned}
$$

1 Our convention for the sign of $[i j]$ follows [4] and is the opposite of the convention in much of the earlier physics literature. In particular, $\left(p_{i}+p_{j}\right)^{2}=\langle i j\rangle[i j]=-\left\langle i\left|p_{j}\right| i\right]$ here. 
The recursion relation of [31] can be written as follows.

$$
\begin{aligned}
& A\left(1^{-}, 2, \ldots,(n-1), n^{+}\right)= \\
& \sum_{i=2}^{n-2} \sum_{h=+,-}\left(A\left(\widehat{1}, 2, \ldots, i,-\widehat{P}_{1, i}^{h}\right) \frac{1}{P_{1, i}^{2}} A\left(+\widehat{P}_{1, i}^{-h}, i+1, \ldots, n-1, \widehat{n}\right)\right),
\end{aligned}
$$

where

$$
\begin{gathered}
\widehat{P}_{1, i}=P_{1, i}+\frac{P_{1, i}^{2}}{\left\langle 1\left|P_{1, i}\right| n\right]} \lambda_{1} \tilde{\lambda}_{n} \\
\widehat{p}_{1}=p_{1}+\frac{P_{1, i}^{2}}{\left\langle 1\left|P_{1, i}\right| n\right]} \lambda_{1} \tilde{\lambda}_{n} \\
\widehat{p}_{n}=p_{n}-\frac{P_{1, i}^{2}}{\left\langle 1\left|P_{n, i}\right| n\right]} \lambda_{1} \tilde{\lambda}_{n} .
\end{gathered}
$$

Here we have chosen the gluons labeled by 1 and $n$ to be the reference gluons.

It was noted in [31] that amplitudes in split helicity configurations are closed under the recursion and that for this case, the sum (2.3) has only two nonzero terms. We turn our attention to such amplitudes in the next section. However, some of the technical points appearing in our calculation below turn out to be generic to the application of (2.3) to any class of amplitudes, and we will comment on such generalizations when appropriate.

\section{Split Helicity Amplitudes and Zigzag Diagrams}

The main result of this note is the following formula for general tree-level split helicity gluon amplitudes:

$$
A\left(1^{-}, \ldots, q^{-},(q+1)^{+}, \ldots, n^{+}\right)=\sum_{k=0}^{\min (q-3, n-q-2)} \sum_{A_{k}, B_{k+1}} \frac{N_{1} N_{2} N_{3}}{D_{1} D_{2} D_{3}} .
$$

Here $A_{k}$ and $B_{k+1}$ respectively range over all subsets of the indices $\{2, \ldots, q-2\}$ and $\{q+1, \ldots, n-1\}$ of cardinality $k$ and $k+1$. In increasing numerical order, the elements are labeled $a_{1}, a_{2}, \ldots, a_{k}$ and $b_{k+1}, \ldots, b_{1}$. There are a total of $\left(\begin{array}{l}n-4 \\ q-2\end{array}\right)$ terms in the sum. The quantities $N$ and $D$ are defined by

$$
\begin{aligned}
& N_{1}=\left\langle 1\left|P_{2, b_{1}} P_{b_{1}+1, a_{1}} P_{a_{1}+1, b_{2}} \cdots P_{b_{k+1}+1, q-1}\right| q\right\rangle^{3}, \\
& N_{2}=\left\langle b_{1}+1 b_{1}\right\rangle\left\langle b_{2}+1 \quad b_{2}\right\rangle \cdots\left\langle b_{k+1}+1 b_{k+1}\right\rangle, \\
& N_{3}=\left[\begin{array}{ll}
a_{1} & a_{1}+1
\end{array}\right] \cdots\left[\begin{array}{ll}
a_{k} & a_{k}+1
\end{array}\right], \\
& D_{1}=P_{2, b_{1}}^{2} P_{b_{1}+1, a_{1}}^{2} P_{a_{1}+1, b_{2}}^{2} \cdots P_{b_{k+1}+1, q-1}^{2}, \\
& D_{2}=F_{q, 1} \bar{F}_{2, q-1}, \\
& D_{3}=\left[2\left|P_{2, b_{1}}\right| b_{1}+1\right\rangle\left\langle b_{1}\left|P_{b_{1}+1, a_{1}}\right| a_{1}\right]\left[a_{1}+1\left|P_{a_{1}+1, b_{2}}\right| b_{2}+1\right\rangle \cdots\left\langle b_{k+1}\left|P_{b_{k+1}+1, q-1}\right| q-1\right]
\end{aligned}
$$


where $F_{x, y}$ is given by

$$
F_{x, y}=\langle x x+1\rangle\langle x+1 x+2\rangle \cdots\langle y-1 y\rangle,
$$

and $\bar{F}_{x, y}$ is given by the same expression but with the inner product $[\cdot \cdot]$.

We find it helpful to illustrate each term of (3.1) by a zigzag diagram, drawn as follows:

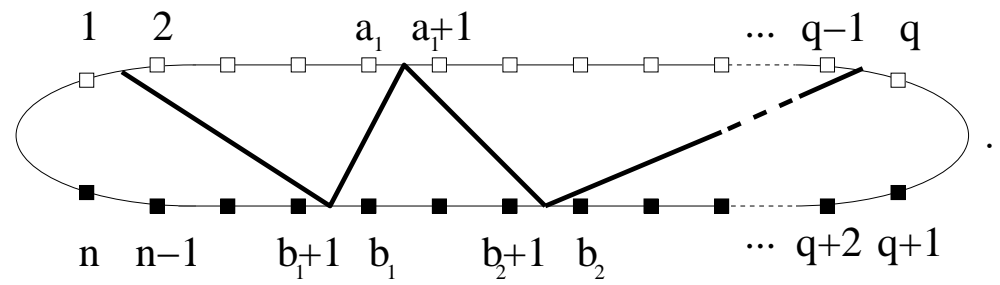

Arrange the gluon indices in clockwise order around a closed curve, with the negative helicities $\{1, \ldots, q\}$ on the top side and the positive helicities $\{q+1, \ldots, n\}$ on the bottom side. A zigzag is a connected collection of non-self-intersecting line segments which begins at $(1,2)$ and ends at $(q-1, q)$, alternating at each step between the top and bottom sides. It is clear that there is a one-to-one correspondence between such zigzag diagrams and choices of the subsets $A_{k}$ and $B_{k+1}$. The line segments in a zigzag diagram are in oneto-one correspondence with the momenta $P_{x, y}$ appearing in the expressions (3.2), and the rule for transforming any given zigzag diagram into a formula is clear from (3.2).

The observation that the line segments correspond to cyclic sums of momenta motivates a relatively simple diagrammatic proof that (3.1) has the correct multi-particle factorization property. In particular, contributions to the $1 / P_{x, y}^{2}$ pole in $A_{q, n-q}$ can only come from those zigzags which contain a line segment that cuts the amplitude so that gluons $(x, x+1, \ldots, y)$ are on one side of the cut.

\section{Examples}

In this section we illustrate the application of (3.1) by means of several examples.

\subsection{MHV amplitudes}

We can view the MHV amplitudes $(q=2)$ as a special case where the zigzag collapses to a point. In this case there are no $P$ 's, so the only factors in (3.2) which contribute are $N_{1}=\langle 12\rangle^{3}$ and $D_{2}=F_{2,1}$. This gives immediately the desired result

$$
A\left(1^{-}, 2^{-}, 3^{+}, \ldots, n^{+}\right)=\overbrace{n}=\frac{\langle 12\rangle^{3}}{\langle 23\rangle\langle 34\rangle \cdots\langle n 1\rangle} .
$$




\section{2. $\overline{\mathrm{MHV}}$ amplitudes}

For $\overline{\mathrm{MHV}}$ amplitudes $(n=q+2)$ there is only a single zigzag diagram,

$$
\begin{aligned}
& A\left(1^{-}, 2^{-}, \ldots, q^{-},(q+1)^{+},(q+2)^{+}\right)= \\
& =\frac{1}{F_{q, 1} \bar{F}_{2, q-1}} \frac{\left\langle 1\left|P_{2, q+1} P_{q+2, q-1}\right| q\right\rangle^{3}}{P_{2, q+1}^{2} P_{q+2, q-1}^{2}} \frac{\langle q+2 q+1\rangle}{\left[2\left|P_{2, q+1}\right| q+2\right\rangle\left\langle q+1\left|P_{q+2, q-1}\right| q-1\right]},
\end{aligned}
$$

which immediately simplifies to the expected result

$$
A\left(1^{-}, 2^{-}, \ldots, q^{-},(q+1)^{+},(q+2)^{+}\right)=\frac{[q+1 q+2]^{3}}{[q+21]\left[\begin{array}{ll}
1 & 2
\end{array}\right] \cdots[q q+1]} .
$$

The zigzag rules treat $\mathrm{MHV}$ and $\overline{\mathrm{MHV}}$ amplitudes differently. More generally, the zigzag rules do not manifestly respect the symmetry

$$
A_{q, n-q}\left(1^{-}, \ldots, q^{-},(q+1)^{+}, \ldots, n^{+}\right)=\overline{A_{n-q, q}\left((q+1)^{-}, \ldots, n^{-}, 1^{+}, \ldots, q^{+}\right)} .
$$

It is clear that one can formulate an alternate set of rules involving zigzags that begin and end on the positive helicity side of the diagram, with some straightforward changes to (3.2). This alternate set of rules might lead to simpler intermediate expressions for amplitudes which have more negative than positive helicity gluons, although the final expression for any amplitude would of course be equal to that obtained from the version of the rules that we presented above.

\subsection{Split helicity next-to-MHV amplitudes}

Next we consider the split helicity next-to-MHV amplitudes $(q=3)$ for arbitrary $n$. In this case there is only one kind of zigzag diagram which contributes, allowing us to immediately write down the result

$$
\begin{aligned}
A\left(1^{-}, 2^{-}, 3^{-}, 4^{+}, \ldots, n^{+}\right) & =\sum_{j=4}^{n-1} \\
& =\frac{1}{F_{3,1}} \sum_{j=4}^{n-1} \frac{\left\langle 1\left|P_{2, j} P_{j+1,2}\right| 3\right\rangle^{3}}{P_{2, j}^{2} P_{j+1,2}^{2}} \frac{\langle j+1 j\rangle}{\left[2\left|P_{2, j}\right| j+1\right\rangle\left\langle j\left|P_{j+1,2}\right| 2\right]} .
\end{aligned}
$$

This formula, which is equivalent to one recently obtained in [34], is noticeably more compact than previously-known expressions for next-to-MHV amplitudes [40,7,10]. 


\subsection{The amplitude $A\left(1^{-}, 2^{-}, 3^{-}, 4^{-}, 5^{+}, 6^{+}, 7^{+}, 8^{+}\right)$}

A compact six-term formula for the eight-particle split helicity next-to-next-to-MHV amplitude $A\left(1^{-}, 2^{-}, 3^{-}, 4^{-}, 5^{+}, 6^{+}, 7^{+}, 8^{+}\right)$was first written down in [30]. It is straightforward to check that this formula is immediately reproduced by summing the following six zigzag diagrams:

$$
\begin{aligned}
& \underbrace{}_{7} \int_{6}^{2}=\frac{1}{F_{4,1} \bar{F}_{2,3}} \frac{\left\langle 1\left|P_{2,5} P_{6,3}\right| 4\right\rangle^{3}}{P_{2,5}^{2} P_{6,3}^{2}} \frac{\langle 65\rangle}{\left[2\left|P_{2,5}\right| 6\right\rangle\left\langle 5\left|P_{6,3}\right| 3\right]}, \\
& V_{8} V_{6}^{2}=\frac{1}{F_{4,1} \bar{F}_{2,3}} \frac{\left\langle 1\left|P_{2,6} P_{7,3}\right| 4\right\rangle^{3}}{P_{2,6}^{2} P_{7,3}^{2}} \frac{\langle 76\rangle}{\left[2\left|P_{2,6}\right| 7\right\rangle\left\langle 6\left|P_{7,3}\right| 3\right]}, \\
& \int_{8}^{2}=\frac{1}{F_{4,1} \bar{F}_{2,3}} \frac{\left\langle 1\left|P_{2,7} P_{8,3}\right| 4\right\rangle^{3}}{P_{2,7}^{2} P_{8,3}^{2}} \frac{\langle 87\rangle}{\left[2\left|P_{2,7}\right| 8\right\rangle\left\langle 7\left|P_{8,3}\right| 3\right]}
\end{aligned}
$$

$$
V_{7}^{2} \underbrace{3}_{6}=\frac{1}{F_{4,1} \bar{F}_{2,3}} \frac{\left\langle 1\left|P_{2,6} P_{7,2} P_{3,5} P_{6,3}\right| 4\right\rangle^{3}}{P_{2,6}^{2} P_{7,2}^{2} P_{3,5}^{2} P_{6,3}^{2}} \frac{\langle 76\rangle \quad[23] \quad\langle 65\rangle}{\left[2\left|P_{2,6}\right| 7\right\rangle\left\langle 6\left|P_{7,2}\right| 2\right]\left[3\left|P_{3,5}\right| 6\right\rangle\left\langle 5\left|P_{6,3}\right| 3\right]}
$$

$V_{7} V_{6}^{2}=\frac{1}{F_{4,1} \bar{F}_{2,3}} \frac{\left\langle 1\left|P_{2,7} P_{8,2} P_{3,5} P_{6,3}\right| 4\right\rangle^{3}}{P_{2,7}^{2} P_{8,2}^{2} P_{3,5}^{2} P_{6,3}^{2}} \frac{\langle 87\rangle \quad[23]}{\left[2\left|P_{2,7}\right| 8\right\rangle\left\langle 7\left|P_{8,2}\right| 2\right]\left[3\left|P_{3,5}\right| 6\right\rangle\left\langle 5\left|P_{6,3}\right| 3\right]}$

$$
V_{8} \int_{6}^{2}=\frac{1}{F_{4,1} \bar{F}_{2,3}} \frac{\left\langle 1\left|P_{2,7} P_{8,2} P_{3,6} P_{7,3}\right| 4\right\rangle^{3}}{P_{2,7}^{2} P_{8,2}^{2} P_{3,6}^{2} P_{7,3}^{2}} \frac{\langle 87\rangle \quad[23] \quad\langle 76\rangle}{\left[2\left|P_{2,7}\right| 8\right\rangle\left\langle 7\left|P_{8,2}\right| 2\right]\left[3\left|P_{3,6}\right| 7\right\rangle\left\langle 6\left|P_{7,3}\right| 3\right]}
$$

We have written out each term carefully in order to emphasize how simple it is to translate each picture into the corresponding formula, but of course several of the terms can be somewhat simplified. 


\section{Proof of the Main Result}

In this section we present an elementary proof of (3.1). We first reduce the quadratic recursion (2.3) to a simpler linear version. This processed version of the recursion relates an amplitude with $q$ negative helicity gluons to amplitudes with only $q-1$ negative helicity gluons. This can be done for any kind of amplitude, but for split helicity amplitudes the resulting recursion turns out to be simple enough that it can easily be solved by induction in the number of negative helicity gluons. The initial condition for the induction is the case $q=2$, which in section 4.1 we showed gives the correct result. In what follows we use the notation

$$
A_{q, n-q}=A_{q, n-q}\left(1^{-}, \ldots, q^{-},(q+1)^{+}, \ldots, n^{+}\right) .
$$

\section{Step 1.}

First we apply the recursion (2.3) to the amplitude $A_{q, n-q}$ with reference momenta $n$ and 1 . There are only two nonvanishing contributions. In each term, one of the amplitudes is a three-gluon vertex and the other is an $n-1$ gluon amplitude. Specifically, we find (after a little simplification) that (2.3) can be cast into the form

$$
A_{q, n-q}=\frac{[n 2]}{[n 1][12]} A_{q-1, n-q}\left(\widehat{2}^{-}, \ldots, \widehat{n}^{+}\right)+\frac{\langle n-11\rangle}{\langle n-1 n\rangle\langle n 1\rangle} A_{q,(n-1)-q}\left(\widehat{1}^{-}, \ldots, \widehat{n-1}^{+}\right),
$$

where the shifted spinors are

$$
\begin{aligned}
\lambda_{\widehat{1}} & =\lambda_{1}, \\
\lambda_{\widehat{2}} & =\lambda_{2}+\frac{[n 1]}{[n 2]} \lambda_{1}, \\
\lambda_{\widehat{n-1}} & =\lambda_{n-1}, \\
\lambda_{\widehat{n}} & =\lambda_{n}+\frac{[12]}{[n 2]} \lambda_{1}, \\
\widetilde{\lambda}_{\widehat{1}} & =\widetilde{\lambda}_{1}+\frac{\langle n-1 n\rangle}{\langle n-11\rangle} \widetilde{\lambda}_{n}, \\
\widetilde{\lambda}_{\widehat{2}} & =\widetilde{\lambda}_{2}, \\
\widetilde{\lambda}_{\widehat{n-1}} & =\widetilde{\lambda}_{n-1}+\frac{\langle n 1\rangle}{\langle n-11\rangle} \widetilde{\lambda}_{n}, \\
\widetilde{\lambda}_{\widehat{n}} & =\widetilde{\lambda}_{n} .
\end{aligned}
$$

Note that we have relabeled the momentum $\widehat{P}$ to $\widehat{2}$ in the first term in (5.2) and to $\widehat{n-1}$ in the second term. This puts (5.2) into a form that can easily be fed back into itself. 


\section{Step 2.}

Next we would like to use (5.2) to express $A_{q, n-q}$ in terms of amplitudes which have strictly less than $q$ negative helicity gluons. We leave the first term in (5.2) alone since it already has $q-1$ negative helicity gluons, but to the second term we apply the result of (5.2) again to rewrite (schematically)

$$
A_{q,(n-1)-q}=A_{q-1,(n-1)-1}+A_{q,(n-2)-q}
$$

We continue applying (5.2) in this manner to strip away $j$ positive helicity gluons from the amplitude. It is very convenient to continue using the first and last gluons (in the order written) as the reference gluons. This process terminates at $j=n-q-1$ since an amplitude with only a single positive helicity gluon vanishes, and we obtain the desired expression

$$
A_{q, n-q}=-\sum_{j=0}^{n-q-2} \frac{\left\langle 1\left|P_{2, n-j-1}\right| 2\right]}{F_{n-j, 1} P_{2, n-j-1}^{2}\left\langle n-j\left|P_{2, n-j-1}\right| 2\right]} A_{q-1,(n-j)-q}\left(\widehat{2}^{-}, \ldots, \widehat{n-j}^{+}\right)
$$

in terms of the shifted spinors

$$
\begin{aligned}
\lambda_{\widehat{2}} & =\frac{P_{n-j, 2} P_{2, n-j-1}|1\rangle}{\left\langle 1\left|P_{2, n-j-1}\right| 2\right]}, \\
\lambda_{\widehat{n-j}} & =\frac{\left.P_{2, n-j-1} \mid 2\right]}{\left\langle 1\left|P_{2, n-j-1}\right| 2\right]}, \\
\widetilde{\lambda}_{\widehat{2}} & =\widetilde{\lambda}_{2}, \\
\widetilde{\lambda}_{\widehat{n-j}} & =P_{2, n-j-1}|1\rangle .
\end{aligned}
$$

It is important to note that the procedure of expressing an amplitude with $q$ negative helicity gluons in terms of amplitudes with only $q-1$ negative helicity gluons works in complete generality. Let us use $A_{n_{1}^{-}, n_{1}^{+}, \ldots, n_{k}^{-}, n_{k}^{+}}$to denote an amplitude in which the first $n_{1}^{-}$gluons have negative helicity, the next $n_{1}^{+}$have positive helicity, and so on. By repeatedly splitting off the last group of positive helicity gluons, in a manner similar to what we did above, it is possible to show that the recursion (2.3) can always be processed into the form

$$
A_{n_{1}^{-}, n_{1}^{+}, \ldots, n_{k}^{-}, n_{k}^{+}}=\sum_{j=0}^{n_{k}^{+}-2}\left[U_{j}(p) A_{\left(n_{1}-1\right)^{-}, n_{1}^{+}, \ldots, n_{k}^{-},\left(n_{k}-j\right)^{+}}+R_{j}(p)\right]
$$


The functions $U_{j}(p)$ are universal functions of the spinors, depending only on the threegluon amplitudes $(++-)$ and $(+--)$. In (5.5) we have determined these functions from the analysis of the special case $k=1$. In contrast, the $R_{j}(p)$ term depends sensitively on the particular class of amplitudes under consideration. It is the happy fact that $R_{j}(p)$ vanishes for split helicity amplitudes that will allow us to solve the recursion (5.5) inductively in this case.

\section{Step 3.}

This step is nothing but convenient bookkeeping: we simply relabel the summation index in (5.5) from $j$ to $b=n-j-1$. After some other minor changes, we have

$$
A_{q, n-q}=\sum_{b=q+1}^{n-1} \frac{\left\langle 1\left|P_{2, b}\right| 2\right]}{F_{b+1,1} P_{b+1,1}^{2}\left\langle b+1\left|P_{b+1,1}\right| 2\right]} A_{q-1,(b+1)-q}\left(\widehat{2}^{-}, \ldots, \widehat{b+1}^{+}\right)
$$

with shifted spinors

$$
\begin{aligned}
\lambda_{\widehat{2}} & =\frac{P_{3, b} P_{b+1,1}|1\rangle}{\left\langle 1\left|P_{2, b}\right| 2\right]}, \\
\lambda_{\widehat{b+1}} & =\frac{\left.P_{2, b} \mid 2\right]}{\left\langle 1\left|P_{2, b}\right| 2\right]}, \\
\widetilde{\lambda}_{\widehat{2}} & =\widetilde{\lambda}_{2} \\
\widetilde{\lambda}_{\widehat{b+1}} & =P_{2, b}|1\rangle .
\end{aligned}
$$

\section{Step 4.}

The aim of our proof is now to show that the expression (3.1) inductively follows from the processed recursion (5.8). Let us start by explaining how the zigzag diagrams which contribute to $A_{q-1,(b+1)-q}$ on the right-hand side of (5.8) lift to zigzag diagrams contributing to $A_{q, n-q}$. For fixed $b \in\{q+1, \ldots, n-1\}$, these diagrams fall into two categories. Either $b_{1}$ (the largest element of the subset $B_{k}$ ) is equal to $b$, or it is less than $b$. In the former case, the lift looks like

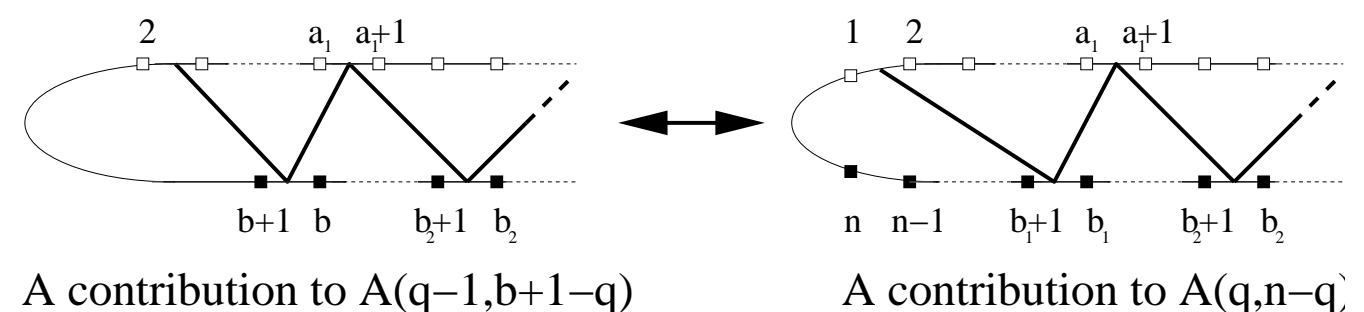


while in the latter case, the lift involves the addition of an extra zigzag (shown here as a dotted line for emphasis)

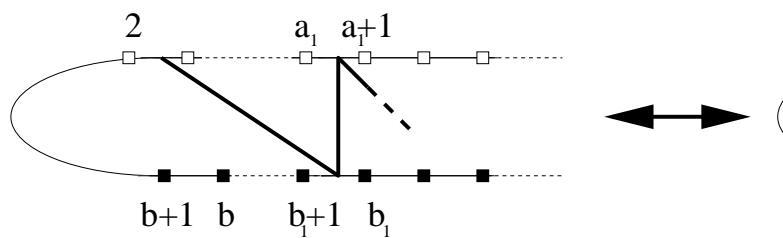

A contribution to $A(q-1, b+1-q)$

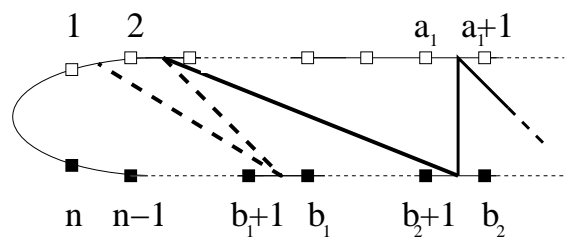

A contribution to $A(q, n-q)$

The reverse map is also clear: a zigzag diagram contributing to $A_{q, n-q}$ comes from the $b=b_{1}$ or the $b=b_{2}$ term in the sum (5.8) depending on whether $a_{1}>2$ or $a_{1}=2$ respectively. We have therefore established a one-to-one correspondence between the zigzag diagrams appearing on the left-hand side of (5.8) and those appearing on the right-hand side. The final step is to establish the quantitative agreement between the two sides.

Step 5a. First consider, for fixed $b \in\{q+1, \ldots, n+1\}$, a zigzag diagram contributing to $A_{q-1,(b+1)-q}$ which has $b_{1}=b$. Note that none of the $P_{x, y}$ appearing carry a single hatted index $(\widehat{2}$ or $\widehat{b+1})$. They always appear together as

$$
\cdots+p_{b}+p_{\widehat{b+1}}+p_{\widehat{2}}+p_{3}+\cdots=\cdots+p_{b}+p_{b+1}+p_{b+2}+\cdots+p_{2}+p_{3}+\cdots,
$$

so we never need to worry about any hats appearing on the $P$ 's. Using (5.9) we find

$$
\begin{aligned}
N_{1}= & \left\langle\widehat{2}\left|P_{3, b} P_{b+1, a_{1}} \cdots P_{b_{k+1}+1, q-1}\right| q\right\rangle^{3}=\left[\frac{\left(P_{3, b}^{2}\right)^{3}}{\left\langle 1\left|P_{2, b}\right| 2\right]^{3}}\right]\left\langle 1\left|P_{2, b} P_{b+1, a_{1}} \cdots P_{b_{k+1}+1, q-1}\right| q\right\rangle^{3}, \\
N_{2}= & \langle\widehat{b+1} b\rangle\left\langle b_{2} b_{2}+1\right\rangle \cdots\left\langle b_{k+1}+1 b_{k+1}\right\rangle \\
= & {\left[\frac{1}{F_{b, b+1}} \frac{\left\langle b\left|P_{2, b}\right| 2\right]}{\left\langle 1\left|P_{2, b}\right| 2\right]}\right]\langle b+1 b\rangle\left\langle b_{2} b_{2}+1\right\rangle \cdots\left\langle b_{k+1}+1 b_{k+1}\right\rangle, } \\
N_{3}= & {\left[a_{1} a_{1}+1\right] \cdots\left[a_{k} a_{k}+1\right], } \\
D_{1}= & P_{q, b_{1}}^{2} P_{b_{1}+1, a_{1}}^{2} P_{a_{1}+1, b_{2}}^{2} \cdots P_{a_{k}+1, b}^{2} P_{b+1,2}^{2} \\
= & {\left[\frac{P_{b+1,2}^{2}}{P_{b+1,1}^{2}}\right] P_{q, b_{1}}^{2} P_{b_{1}+1, a_{1}}^{2} P_{a_{1}+1, b_{2}}^{2} \cdots P_{a_{k}+1, b}^{2} P_{b+1,1}^{2}, } \\
D_{2}= & F_{q, \widehat{2}} \bar{F}_{3, q-1}=\left[\frac{\left\langle b\left|P_{2, b}\right| 2\right]}{\left\langle 1\left|P_{2, b}\right| 2\right]^{2}} P_{3, b}^{2} \frac{1}{F_{b, 1}[23]}\right] F_{q, 1} \bar{F}_{2, q-1}, \\
D_{3}= & {\left[3\left|P_{3, b}\right| \widehat{b+1}\right\rangle\left\langle b\left|P_{b+1, a_{1}}\right| a_{1}\right] \cdots\left\langle b_{k+1}\left|P_{b_{k+1}+1, q-1}\right| q-1\right] } \\
= & {\left[\frac{P_{3, b}^{2}[23]}{\left\langle 1\left|P_{2, b}\right| 2\right]\left\langle b+1\left|P_{b+1,2}\right| 2\right]}\right]\left[2\left|P_{3, b}\right| b+1\right\rangle\left\langle b\left|P_{b+1, a_{1}}\right| a_{1}\right] \cdots\left\langle b_{k+1}\left|P_{b_{k+1}+1, q-1}\right| q-1\right] . }
\end{aligned}
$$


In each term we have assembled in brackets all of the extra factors which are not present in the corresponding contribution to the zigzag diagram on the right-hand side of (5.10). When we take the ratio $N_{1} N_{2} N_{3} / D_{1} D_{2} D_{3}$ we find that these extra factors combine into

$$
\frac{F_{b+1,1} P_{b+1,1}^{2}\left\langle b+1\left|P_{b+1,1}\right| 2\right]}{\left\langle 1\left|P_{2, b}\right| 2\right]},
$$

which precisely cancels the factor appearing in (5.8). This establishes the quantitative agreement between the diagrams shown in (5.10).

Step 5b. Finally, we consider the case shown in (5.11), with a zigzag diagram contributing to $A_{q-1,(b+1)-q}$ such that $b_{1}<b$. In this case the analysis is simpler, since only $N_{1}$ and $D_{2}$ develop extra factors relative to what one would expect from looking at the second diagram in (5.11):

$$
\begin{aligned}
N_{1} & =\left\langle\widehat{2}\left|P_{3, b_{1}} P_{b_{1}+1, a_{1}} \cdots P_{b_{k+1}+1, q-1}\right| q\right\rangle^{3} \\
& =\left[\frac{1}{\left\langle 1\left|P_{2, b}\right| 2\right]^{3}}\right]\left\langle 1\left|P_{2, b_{1}} P_{b_{1}+1, a_{1}} \cdots P_{b_{k+1}+1, q-1}\right| q\right\rangle^{3}, \\
D_{2} & =F_{q, \widehat{2}} \bar{F}_{3, q-1}=\left[-\frac{\left\langle b\left|P_{2, b}\right| 2\right]}{\left\langle 1\left|P_{2, b}\right| 2\right]^{2}} P_{3, b}^{2} \frac{1}{F_{b, 1}[23]}\right] F_{q, 1} \bar{F}_{2, q-1} .
\end{aligned}
$$

Note that $N_{1}$ has grown the two additional legs of the zigzag, shown as dotted lines in (5.11). Combining the factors in brackets with the factors appearing explicitly in (5.8) gives

$$
\frac{\langle b+1 b\rangle[23]}{P_{3, b}^{2} P_{b+1,1}^{2}\left[2\left|P_{2, b}\right| b\right\rangle\left\langle b+1\left|P_{b+1,1}\right| 2\right]},
$$

which are precisely the correct extra factors needed to account for the addition of the dotted zigzag.

\section{Acknowledgments}

We would like to thank Z. Bern and F. Cachazo for helpful discussions and L. Dixon for pointing out typos in equations in sections 3 and 4.4 of the previous version. This research was supported in part by the Department of Energy under Grant No. DE-FG0291ER40671 (RR) and by the National Science Foundation under Grants Nos. PHY99-07949 (MS, AV) and PHY-0070928 (RB, BF). 


\section{References}

[1] S. J. Parke and T. R. Taylor, "An Amplitude For N Gluon Scattering," Phys. Rev. Lett. 56, 2459 (1986).

[2] M. L. Mangano, S. J. Parke and Z. Xu, "Duality And Multi-Gluon Scattering," Nucl. Phys. B 298, 653 (1988).

[3] F. A. Berends and W. T. Giele, "Recursive Calculations For Processes With N Gluons," Nucl. Phys. B 306, 759 (1988).

[4] E. Witten, "Perturbative gauge theory as a string theory in twistor space," Commun. Math. Phys. 252, 189 (2004) [arXiv:hep-th/0312171].

[5] R. Roiban, M. Spradlin and A. Volovich, "A googly amplitude from the B-model in twistor space," JHEP 0404, 012 (2004) arXiv:hep-th/0402016.

[6] R. Roiban and A. Volovich, "All conjugate-maximal-helicity-violating amplitudes from topological open string theory in twistor space," Phys. Rev. Lett. 93, 131602 (2004) arXiv:hep-th/0402121.

[7] F. Cachazo, P. Svrcek and E. Witten, "MHV vertices and tree amplitudes in gauge theory," JHEP 0409, 006 (2004) arXiv:hep-th/0403047.

[8] C. J. Zhu, "The googly amplitudes in gauge theory," JHEP 0404, 032 (2004) arXiv:hep-th/0403115.

[9] R. Roiban, M. Spradlin and A. Volovich, "On the tree-level S-matrix of Yang-Mills theory," Phys. Rev. D 70, 026009 (2004) [arXiv:hep-th/0403190].

[10] D. A. Kosower, "Next-to-maximal helicity violating amplitudes in gauge theory," Phys. Rev. D 71, 045007 (2005) arXiv:hep-th/0406175.

[11] S. Gukov, L. Motl and A. Neitzke, "Equivalence of twistor prescriptions for super Yang-Mills," arXiv:hep-th/0404085.

[12] J. B. Wu and C. J. Zhu, "MHV vertices and scattering amplitudes in gauge theory," JHEP 0407, 032 (2004) arXiv:hep-th/0406085].

[13] I. Bena, Z. Bern and D. A. Kosower, "Twistor-space recursive formulation of gauge theory amplitudes," Phys. Rev. D 71, 045008 (2005) arXiv:hep-th/0406133.

[14] Z. Bern, D. Forde, D. A. Kosower and P. Mastrolia, "Twistor-inspired construction of electroweak vector boson currents," Phys. Rev. D 72, 025006 (2005) arXiv:hep$\mathrm{ph} / 0412167$.

[15] R. J. Eden, P. V. Landshoff, D. I. Olive and J. C. Polkinghorne, The Analytic S-Matrix, Cambridge University Press, 1966.

[16] W. T. Giele, E. W. N. Glover and D. A. Kosower, "Higher order corrections to jet crosssections in hadron colliders," Nucl. Phys. B 403, 633 (1993) arXiv:hep-ph/9302225.

[17] Z. Kunszt, A. Signer and Z. Trocsanyi, "Singular terms of helicity amplitudes at one loop in QCD and the soft limit of the cross-sections of multiparton processes," Nucl. Phys. B 420, 550 (1994) arXiv:hep-ph/9401294. 
[18] I. Bena, Z. Bern, D. A. Kosower and R. Roiban, "Loops in twistor space," Phys. Rev. D 71, 106010 (2005) arXiv:hep-th/0410054.

[19] F. Cachazo, "Holomorphic anomaly of unitarity cuts and one-loop gauge theory amplitudes," arXiv:hep-th/0410077.

[20] R. Britto, F. Cachazo and B. Feng, "Computing one-loop amplitudes from the holomorphic anomaly of unitarity cuts," Phys. Rev. D 71, 025012 (2005) arXiv:hepth/0410179.

[21] R. Britto, F. Cachazo and B. Feng, "Generalized unitarity and one-loop amplitudes in $\mathcal{N}=4$ super-Yang-Mills," Nucl. Phys. B 725, 275 (2005) arXiv:hep-th/0412103.

[22] Z. Bern, L. J. Dixon and D. A. Kosower, "All next-to-maximally helicity-violating one-loop gluon amplitudes in $\mathcal{N}=4$ super-Yang-Mills theory," Phys. Rev. D 72, 045014 (2005) arXiv:hep-th/0412210.

[23] Z. Bern, L. J. Dixon, D. C. Dunbar and D. A. Kosower, "One loop $n$ point gauge theory amplitudes, unitarity and collinear limits," Nucl. Phys. B 425, 217 (1994) arXiv:hep-ph/9403226.

[24] Z. Bern, L. J. Dixon, D. C. Dunbar and D. A. Kosower, "Fusing gauge theory tree amplitudes into loop amplitudes," Nucl. Phys. B 435, 59 (1995) arXiv:hep-ph/9409265.

[25] Z. Bern, L. J. Dixon and D. A. Kosower, "One-loop amplitudes for $e^{+} e^{-}$to four partons," Nucl. Phys. B 513, 3 (1998) arXiv:hep-ph/9708239.

[26] Z. Bern, L. J. Dixon and D. A. Kosower, "Unitarity-based techniques for one-loop calculations in QCD," Nucl. Phys. Proc. Suppl. 51C, 243 (1996) arXiv:hep-ph/9606378.

[27] Z. Bern and A. G. Morgan, "Massive Loop Amplitudes from Unitarity," Nucl. Phys. B 467, 479 (1996) arXiv:hep-ph/9511336.

[28] Z. Bern, L. J. Dixon and D. A. Kosower, "Progress in one-loop QCD computations," Ann. Rev. Nucl. Part. Sci. 46, 109 (1996) arXiv:hep-ph/9602280.

[29] Z. Bern, V. Del Duca, L. J. Dixon and D. A. Kosower, "All non-maximally-helicityviolating one-loop seven-gluon amplitudes in $\mathcal{N}=4$ super-Yang-Mills theory," Phys. Rev. D 71, 045006 (2005) arXiv:hep-th/0410224.

[30] R. Roiban, M. Spradlin and A. Volovich, "Dissolving $\mathcal{N}=4$ loop amplitudes into QCD tree amplitudes," Phys. Rev. Lett. 94, 102002 (2005) arXiv:hep-th/0412265.

[31] R. Britto, F. Cachazo and B. Feng, "New recursion relations for tree amplitudes of gluons," Nucl. Phys. B 715, 499 (2005) [arXiv:hep-th/0412308.

[32] R. Britto, F. Cachazo, B. Feng and E. Witten, "Direct proof of tree-level recursion relation in Yang-Mills theory," Phys. Rev. Lett. 94, 181602 (2005) arXiv:hep-th/0501052.

[33] A. Hodges, "Twistor diagram recursion for all gauge-theoretic tree amplitudes," arXiv:hep-th/0503060.

[34] M. Luo and C. Wen, "Recursion relations for tree amplitudes in super gauge theories," JHEP 0503, 004 (2005) arXiv:hep-th/0501121. 
[35] M. Luo and C. Wen, "Compact formulas for all tree amplitudes of six partons," Phys. Rev. D 71, 091501 (2005) arXiv:hep-th/0502009.

[36] J. Bedford, A. Brandhuber, B. Spence and G. Travaglini, "A recursion relation for gravity amplitudes," Nucl. Phys. B 721, 98 (2005) arXiv:hep-th/0502146.

[37] F. Cachazo and P. Svrcek, "Tree level recursion relations in general relativity," arXiv:hep-th/0502160.

[38] Z. Bern, L. J. Dixon and D. A. Kosower, "On-shell recurrence relations for one-loop QCD amplitudes," Phys. Rev. D 71, 105013 (2005) arXiv:hep-th/0501240.

[39] L. J. Dixon, "Calculating scattering amplitudes efficiently," arXiv:hep-ph/9601359.

[40] D. A. Kosower, "Light Cone Recurrence Relations For QCD Amplitudes," Nucl. Phys. B 335, 23 (1990). 